\title{
Fontes teóricas sobre representações sociais: um perfil bibliométrico de textos citados em periódicos científicos nacionais da área de Saúde
}

Guido Rummler

\author{
Introdução
}

Pode-se aplicar à maioria das áreas da Saúde que abordam questões de interface com as Ciências Humanas e Sociais, o que escreve Carvalho (2003) referindo-se à sua área profissional, a enfermagem: precisamos tanto das bases e dos métodos das ciências nomotéticas (ciências da natureza e das leis cientificas) como necessitamos dos requisitos e regras das ciências ideográficas (as que tratam das representações das idéias e da análise da linguagem).

Possivelmente tal percepção constitua um dos suportes à transição da pesquisa científica em Saúde de uma polarização metodológica quantitativa para uma convivência com paradigmas qualitativos. Assim, é desejável, e parece factível, que das aproximações entre as respectivas metodologias conjuguem-se abordagens que permitam alcançar entendimentos cada vez menos fragmentados das realidades estudadas. Entretanto, convive-se ainda com aspectos extremos, como mencionados por Briceño-León (1995), de que, na área das ciências sociais e saúde, "existe uma diferença fundamental na forma de assumir o objeto de estudo", havendo grupos, até mesmo de áreas oficiais, que "fazem trabalhos minuciosos de coleta de informações, mas não os interpretam a partir de uma perspectiva teórica"; e, por outro lado, "há grupos universitários mais afeitos à teoria, sem pesquisa empírica". Como possíveis causas a primeira situação, diz ainda aquele autor que, "a teoria existe, mas não é levada em consideração, seja devido às limitações de formação, seja pelas urgências de um trabalho prático" (p.36).

Mas, por outro lado. é pela aproximação ou vínculo profissional/universidade que se verifica a produção de conhecimentos gerados pela aplicação de paradigmas metodológicos teoricamente fundamentados, embora, porque nem sempre são levados em conta na aplicação de práticas que pretendem solucionar as questões que os desencadearam, possa tal conduta constituir-se numa terceira situação, tão lastimável quanto as duas antes citadas.

Rummler e Spínola (2005), analisando um amplo elenco de trabalhos empíricos da área de Saúde, que abordam dados qualitativos por paradigmas convencionais ou por outros de desenvolvimento mais recente, verificaram que $12,8 \%$ dessas pesquisas focalizavam

${ }^{1}$ Graduado em Ciências Biológicas; pós-doutorado em Saúde Pública/Metodologia de Pesquisa; professor, Universidade Estadual de Feira de Santana, UEFS. Feira de Santana, BA. <rummler@uefs.br>

Universidade Estadual de Feira de Santana,UEFS - Departamento de Saúde

BR 116, Km 3

Feira de Santana, Ba

44.031-460 
representações sociais (RS) fundamentando-se na Teoria das Representações Sociais (TRS). Tal proporção pode ser considerada significativa, uma vez que a inauguração dessa teoria remonta a apenas quatro décadas. Relativamente a esse caminho metodológico é oportuno mencionar que, de acordo com Sá (1996), representações sociais (RS) designam tanto um conjunto de fenômenos quanto o conceito que os engloba e a teoria construída para explicá-los.

Várias tentativas para expressar um conceito de RS encontram-se na literatura. Franco (2004) parte da premissa de que RS "são elaborações mentais construídas socialmente, a partir da dinâmica que se estabelece entre a atividade psíquica do sujeito e o objeto do conhecimento. Relação que se dá na prática social e história da humanidade e que se generaliza pela linguagem" (p.171) Ou ainda, como expressam Cardoso e Arruda (2003), a RS é definida como uma forma de conhecimento do "senso comum", que está diretamente relacionada à maneira como as pessoas interpretam os conhecimentos veiculados socialmente. Minayo (2004), sintetizando colocações de vários autores, identifica "as representações sociais enquanto senso comum, idéias, imagens, concepções e visão de mundo que os atores sociais possuem sobre a realidade, são um material importante para a pesquisa no interior das Ciências Sociais" (p.173). Por outro lado, o caráter da inter e transdisciplinaridade, intrínseco às representações sociais, conforme discute Spink (1993), situando-as na interface dos fenômenos individual e coletivo, justifica suas diversas abordagens e aplicações, cabendo-lhes também articulação com áreas da Saúde, na focalização de diversos campos temáticos, como os identificados em trabalhos anteriores (Rummler \& Spínola, 2005).

Segundo Arruda (2002b), "La Psychanalyse, son image, son public", de Serge Moscovici, publicada em 1961, constitui a obra seminal que contém a matriz da Teoria das Representações Sociais. Como foram, daí em diante, entendidos, elaborados, explicados e desenvolvidos os constituintes dessa teoria corresponde certamente a uma produção literária tanto do próprio Moscovi como de diversos outros estudiosos, além dos já citados.

A identificação de quais dessas fontes os pesquisadores empíricos da área da Saúde têm se valido para embasar seus trabalhos, constitui um dos aspectos que, preliminarmente, se pretende verificar no presente estudo. A resposta a essa interrogação, apresentada na forma de uma listagem em que as obras são identificadas pela correspondente referência bibliográfica, pode constituir elemento útil a eventuais interessados no trabalho de busca por fontes sobre TRS.

Numa segunda etapa deste estudo, é feita uma análise bibliométrica referente ao conjunto das fontes identificadas. Deve-se ressaltar, entretanto, que, por essa análise quantitativa, não se pretende atribuir àquelas fontes, ou a seus autores, conotações de ordem valorativa quanto à respectiva contribuição, na medida que tão-somente busca-se estabelecer aspectos relacionados à cronologia, quantidade de fontes, diversidade de autores e freqüência com que pesquisadores de áreas da Saúde têm a elas recorrido na elaboração de trabalhos de base empírica, em tempos recentes.

\section{Metodologia}

Trata-se de um estudo exploratório, descritivo, baseado em análise de citações e abordagem bibliométrica. Foi desenvolvido com base em artigos contendo pesquisa empírica fundamentada na Teoria das Representações Sociais, obtidos por meio de rastreamento feito em revistas de cobertura de áreas de Ciências da Saúde. Os procedimentos de maior relevância adotados para sua execução foram:

- identificação na lista de periódicos nacionais indexados pelo sistema Lilacs (Bireme, 2003) dos títulos referentes às áreas pertencentes, segundo a Capes (http://www.capes.gov.br/export/ sites/capes/download/avaliacao/areasconhecimento.doc), a Ciências da Saúde, exceto Medicina. A seleção dos periódicos de Saúde Coletiva foi feita com base em estudo de Rummler e Spínola (2003), que aponta revistas cujas denominações se referem à Medicina, Nutrição, Educação, etc., mas são também referidas à Saúde Coletiva. A identificação de periódicos que cobrem as áreas de Odontologia, Farmácia, Enfermagem, Fisioterapia e Terapêutica Ocupacional e Educação Física, foi feita com base na indicação do título e/ou explicitação editorial; 
- busca a fascículos editados entre 2002 e 2004 dos 76 títulos selecionados, seja em bibliotecas seja por tentativas endereçadas às próprias revistas;

- exame de 258 fascículos de 46 periódicos, cujo acesso foi possível estabelecer, e nos quais foram identificados 3.408 artigos de pesquisa empírica;

- identificação, dos artigos que constituem pesquisa baseada na TRS;

- identificação das fontes de origem das citações de ordem teórica sobre representações sociais, utilizadas nesses trabalhos;

- listagens das referências bibliográficas dessas fontes, normalizadas segundo a Associação Brasileira de Normas Técnicas - ABNT (2002), com base nos elementos de identificação, constantes das referências apresentadas pelos artigos estudados;

- quantificação dessas fontes e sua distribuição proporcional no conjunto de artigos analisados; estabelecimento de relações entre autor/número de obras e freqüência de citação, assim como sua distribuição cronológica quanto à época de publicação.

Os 45 artigos baseados na Teoria das Representações Sociais existentes no universo investigado encontram-se publicados nos seguintes periódicos:

Acta Paulista de Enfermagem, v. 16, n. 2 e 4, 2003; Cadernos de Saúde Pública, v. 19, Supl. 2, 2003; v. 20, n. 1, 2004; v. 20, n. 4, 2004; Cadernos de Saúde Coletiva, v. 10, n. 1 e 2, 2002; v. 11, n. 2, 2003; Ciência e Saúde Coletiva, v. 8, n. 3 e 4, 2003; v. 9, n. 1, 2 e 3, 2004; Cogitare Enfermagem, v. 6, n. 1, 2001; Escola Anna Nery Revista de Enfermagem, v. 7, n. 3, 2003; História, Ciências, Saúde - Manguinhos, v. 10, supl. 1, 2003; Interface - Comunicação, Saúde, Educação, v. 7, n. 12, 2003; Paidéia, v. 11, n. 21, 2001; v. 12, n. 22, 2002; REME Revista Mineira de Enfermagem, v. 6, n. 1/2, 2002; Revista Baiana de Enfermagem, v. 15, n. 1/2, 2002; Revista Brasileira de Epidemiologia, v. 6, n. 4, 2003; Revista Brasileira de Saúde Materno Infantil, v. 3, n. 3, 2003; Revista da Escola de Enfermagem da USP, v. 36, n. 2 e 4, 2002; v. 37, n. 2 e 4, 2003; Revista Enfermagem UERJ, v. 11, n. 2 e 3, 2003; Revista de Nutrição, v. 17, n. 1, 2004; Revista de Saúde Pública, v. 38, n. 3, 2004; Revista LatinoAmericana de Enfermagem, v. 11, n. 3, 4, 5 e 6, 2003; Revista Paulista de Enfermagem, v. 22, n. 1, 2003.

\section{Resultados}

Nos 45 trabalhos empíricos lastreados na Teoria das Representações Sociais, foram identificadas 138 referências bibliográficas de textos teóricos sobre a TRS. Essas referências correspondem a 69 textos envolvendo 49 autores. Cada fonte encontra-se identificada na Tabela 1, seguida da indicação da quantidade de artigos em que é citada. Cada fonte tem entrada apenas uma vez nessa tabela, mesmo que tenha diferentes anos de edição.

Tabela 1. Textos de referência da Teoria das Representações Sociais e respectivo número de artigos (n) em que foram utilizados.

\section{Referências}

ABRIC, Jean Claude. Prefácio. In: SÁ, C. P. Núcleo central das representações sociais. Petrópolis (RJ): Vozes, 1996.

Estudos interdisciplinares de representação social. Goiânia: AB, 1998, 2000.

ABRIC, Jean Claude. L'approche structurelle des représentations sociales: developpements récents. Psychologie \& Societé, n. 4, p. 81-103, 2002.

ABRIC, Jean Claude. O estudo experimental das representações sociais. In: JODELET, Denise. (Org.). As Representações Sociais. Rio de Janeiro: EDUERJ, 2001.

ABRIC, Jean Claude. Pratiques sociales et représentations. Paris: Presses Universitaires de France, 1994, 1997.

ABRIC, Jean Claude. Coopération, compétition et représentations sociales. Cousset: DelVal, 1987. 
AIELLO-VAISBERG, Tania Maria José. O uso de procedimentos projetivos na pesquisa de representações sociais: 1 projeção e transicionalidade. São Paulo: Instituto de Psicologia da USP, 1995. (Mimeogr.)

ALVES, Paulo César; RABELO, Miriam Cristina. Repensando os estudos sobre representações sociais e práticas em saúde/ doença. In: ALVES, P.C.; RABELO, M.C. (Org.) Antropologia da saúde: traçando identidade e explorando fronteiras. Rio de Janeiro: Fiocruz/Relume Dumará, 1998.

ARRUDA, Angela. A mudança e representação social. Temas em psicologia. v.8, n.3, p. 241-247, 2000.

ARRUDA, Angela. Novos significados da saúde e as representações sociais. Cadernos de Saúde Coletiva, v. 10, n.2, p.215-227, 2002.

BREAKWELL, Glynis Marie; CANTER, David V. Empirical approaches to social representations. Oxford: Oxford University Press, 1993.

CLÉMENCE, Alain; LORENZI-CIOLDI, Fábio. (Org.). Représentations sociales et analyses de données. Grenoble:

Presse Universitaire de Grenoble, 1992.

FARR, Robert M. Representações sociais: a teoria e sua história. In: GUARESCHI, Pedrinho; JOVCHELOVITCH, Sandra

(Org.). Textos em representação social. Petrópolis: Vozes, 1994.

FLAMENT, Claude. Structure, dynamique et transformation des représentations sociales. In: ABRIC, Jean Claude. Pratiques

sociales et représentations. Paris: Presses Universitaires de France, 1994.

FLAMENT, Claude. Aspects périphériques des représentations sociales. In: GUIMELLI, Christian. (Org.). Structures et

transformations des représentations sociales. Lausanne: Delachaux et Niestlé, 1994.

FLAMENT, Claude. Aspects péripheriques des représentations sociales. In: GUIMELLI, Christian. (Org.). Structures et

transformations des représentations sociales. Neuchâtel: Delachaux et Niestlé, 1986.

FLAMENT, Claude. Estrutura e dinâmica das representações sociais. In: JODELET, Denise (Org.). As representações sociais. Rio de Janeiro: EDUERJ, 2001.

FLAMENT, Claude. L'analyse de similitude: une technique pour les recherches sur les représentations sociales. Cahier de Psychologie Cognitive, v. 1, n. 4, p. 375-95, 1981.

FLAMENT, Claude. L'analyse de similitude: une technique pour les recherches sur les représentations sociales. In: DOISE,

W.; PALMONARI, A. (org.). L'etude des représentations sociales. Paris: Delachaux et Niestlé, 1986.

GUARESCHI, Pedrinho; JOVCHELOLOVITH, Sandra (orgs.). Textos em Representações Sociais. 2. ed. Petrópolis (RJ): Vozes, 1995.

GUIMELLI, Christian (Org.). Structures et transformations des représentations sociales. Neuchâtel: Delachaux et Niestlé, 1986.

HENRY, P.; MOSCOVICI, Serge. Problèmes de l'analyse de contenu. Langages, v. 2, n.11, p. 36-60, 1968.

HERZLICH, Claudine. A problemática da representação social e sua utilidade no campo da doença. Physis Rev Saúde

Coletiva, v. 1, n.2, p. 23-36, 1991.

JODELET, Denise. Folies et representations sociales. Paris: PUF, 1989.

JODELET, Denise. La representación social: fenómenos, concepto y teoría. In: MOSCOVICI, Serge. (Org). Psicología

Social II. Pensamiento y vida social. Psicologia Social y problemas sociales. Barcelona (Es): Paidós, 1986, 1998.

JODELET, Denise. Representação Social: fenômenos, conceitos e teoria. Rio de Janeiro (RJ): Fundação Getúlio Vargas, 1988.

JODELET, Denise. Representaciones sociales: contribución a un saber sociocultural sin fronteras. In: JODELET, Denise;

GUERRERO TAPIA, Alfredo (Org.). Develando la cultura. Estudios en Representaciones sociales. México: UNAM, 2000.

JODELET, Denise. Representações sociais. Rio de Janeiro: EDUERJ, 2002.

JODELET, Denise. As representações sociais: um domínio em expansão. In: JODELET, Denise (Org.). As representações sociais. Rio de Janeiro, EDUERJ, 2001.

JODELET, Denise. Représentation sociale: phenomenos, concept et theorie. In: MOSCOVICI, Serge. (Org.) Psicologie

Sociale. Paris: PUF, 1984.

JODELET, Denise. Représentations Sociales: un domaine en expansion. In: JODELET, Denise (Org.), Les Représentations sociales. Paris: Puf, 1988.

JODELET, Denise. Representation Sociale: un domaine en expansion. In: MOSCOVICI, Serge. (Ed). Les Représentations

Sociales. Paris: Presses Universitaires de France, 1989 
JODELET, Denise. Representação Social: fenômeno, conceito e teoria. In: MOSCOVICI, Serge. Psycologie Sociale. 2. ed. Paris: PUF, 1989.

JOVCHELOVITCH, Sandra; GUARESCHI, Pedrinho. Textos em representações sociais. Petrópolis (RJ): Vozes, 1994.

LANE, Silvia Tatiana Mourer. Linguagem, pensamento e representações sociais. In: LANE, Silvia Tatiana Mourer; CODO, Wanderley (Org). Psicologia social: o homem em movimento. 2. ed. São Paulo (SP): Brasiliense, 1985.

LANE, Silvia Tatiana Mourer. Usos e abusos do conceito de representação social. In: JOVCHELOVITCH, Sandra;

GUARESCHI, Pedrinho (Org.) Textos em representações sociais. Petrópolis: Vozes, 1994.

LANE, Silvia Tatiana Mourer. Usos e abusos do conceito de representação social. In: SPINK, Mary Jane P. (Org.). 0

conhecimento no cotidiano: as representações sociais na perspectiva da psicologia social. São Paulo (SP): Brasiliense, 1993.

MADEIRA, Margot Campo de. Linguagem e representações sociais: quando a vivência se torna palavra. In: MADEIRA,

Margot Campo de (Org.). Representações sociais e educação: algumas reflexões. Natal: EDUFRN, 1998.

MADEIRA, Margot Campo de. Representações sociais e educação: importância teórico-metodológica de uma relação. In: MOREIRA, Antonia Silva Paredes (Org.). Representações sociais: teoria e prática. João Pessoa: Universitária, 2001. MINAYO, M. C. S. O conceito de representações sociais dentro da sociologia clássica. In: JOCHELOVITCH, Sandra; GUARESHI, Pedrinho (Org). Texto em representações sociais. Rio de Janeiro: Vozes, 1994.

MOLINER, Pascal. Images et représentations sociales. Grenoble (Fr): PUG. 1996.

MOLINER, Pascal. Les méthodes de répérage et d'identification du noyau des représentations sociales. In: GUIMELLI,

Christian (Org.). Structures et transformations des représentations sociales. Lausanne: Delachaux et Niestlé, 1994.

MOSCOVICI, Serge. Notes towards a description of social representation in retrospect and prospect. European Journal of Social Psychology, n.18, p. 211-250, 1998.

MOSCOVICI, Serge. A representação social da psicanálise. Rio de Janeiro: Zahar, 1978.

MOSCOVICl, Serge. Das representações coletivas às representações sociais: elementos para uma história. In: JODELET,

Denise (Org). As Representações Sociais. Rio de Janeiro: Ed. UERJ, 2001.

MOSCOVICI, Serge. On social representations. In: FORGAS, Joseph P. (Ed.) Social cognitions perspectives on

everyday understanding. New York (DC): Academic Press, 1981.

MOSCOVICI, Serge. The history and actuality of social representations. In: FLICK, Uwe (Org.). The Psychology of the

Social. Cambridge: University Press, 1999.

MOSCOVICl, Serge. The phenomenon of social representations. In: FARR, Robert M.; MOSCOVICI, Serge (Ed.). Social

representations. Cambridge: University Press, 1984.

OLIVEIRA, Denise Cristina; MARQUES, S. C.; GOMES, A. M. T.; TEIXEIRA, Maria Cristina Triguero Veloz; AMARAL,

Mariana Almeida do. Análise das evocações livres: uma técnica de análise estrutural das representações sociais. In:

PAREDES, Antonia Silva. Metodologia de estudo das representações sociais. João Pessoa e Lisboa: Editora UBPB/

Portugal, 2003.

OLIVEIRA, Denise Cristina; SIQUEIRA, A. A. F.; ALVARENGA, Augusta Tereza de. As práticas sociais em saúde: uma

releitura à luz das representações sociais. In: MOREIRA, Antonia Silva Paredes; OLIVEIRA, Denise Cristina (Org.).

Estudos interdisciplinares de representação social. Goiânia: AB, 1998.

OLIVEIRA, Fátima O.; WERBA, Graziela Cucchiarelli. Representações sociais. In: STREY, Marlene Neves et al.

Psicologia social contemporânea. Petrópolis, Rio de Janeiro: Vozes, 2002.

QUEIROZ, Marcos S. Representações sociais: uma perspectiva multidisciplinar em pesquisa qualitativa. In: BARATA, Rita

Barradas; BRICEÑO-LÉON, Roberto (Orgs.) Doenças endêmicas. Abordagens sociais, culturais e comportamentais. Rio de Janeiro: Editora Fiocruz, 2000.

SÁ, Celso Pereira de. Núcleo central das representações sociais. Petrópolis: Vozes, 1996.

SÁ, Celso Pereira de. Representações sociais: o conceito e o estado atual da teoria; In: SPINK, Mary Jane P. (Ed). O conhecimento no cotidiano. São Paulo: Brasiliense, 1993, 1995.

SÁ, Celso Pereira de. A construção do objeto de pesquisa em Representações Sociais. Rio de Janeiro: EdUERJ, 1998.

SCHUTZ, Alfred. Fenomenologia e representações sociais. Rio de Janeiro: Zahar, 1979. 
SOUZA FILHO, Edson Alves de. A análise de representações sociais. In: SPINK, Mary Jane P. (Org). O conhecimento no 1 cotidiano. As representações sociais na perspectiva da psicologia social. São Paulo (SP): Editora Brasiliense, 1993.

SPINK, Mary Jane P. Desvendando as teorias implícitas: uma análise das representações sociais. In: DUVEEN, Gerard et al. 1

Textos e representações sociais. Petrópolis: Vozes, 1997.

SPINK, M.J. O conhecimento no cotidiano: as representações sociais na perspectiva da psicologia social. São Paulo:

Brasiliense, 1993a, 1995

SPINK, Mary Jane P. O conceito de representação social na abordagem psicossocial. Cadernos de Saúde Pública, v.9, n.3, 1 p. 300-308, 1993.

VAISBERG, Tania Maria José Aiello. O uso dos procedimentos projetivos na pesquisa de representações sociais: projeção e transicionalidade. [tese] São Paulo (SP): Instituto de Psicologia USP; 1995.

VERGĖS, Pierre. Approche du noyau central: propriété quantitatives et structurales. In: GUIMELLI, Christian (Org.).

Structures et transformations des représentations sociales. Lausanne: Delachaux et Niestlé, 1994.

VERGĖS, Pierre. Ensemble de programmes permettant l'ánalyse des évocations: Mannuel versoin 2.00. Laboratoire

Méditerranée en Sociologie, Aix-en-Provence. 2000.

VERGÈS, Pierre. EVOC - Ensemble des programmes permettant l'ánalyse des evocations: Mannuel d'utilysateur. Aix-en-

Provence (Fr): LAMES-CNRS, 1999.

VERGĖS, Pierre. L'evocation de l'argent: une méthode pour la definitionn du noyau central d'une représentation. Bulletin de

Psychologie, v. 45, p. 203-209, 1992.

VERGÈS, Pierre. Une possible methodologie pour l'approche des répresentations économiques. Communication-

Information, v.6, n.23, p.375-398, 1984.

WAGNER, Wolfgang. Descrição, explicação e método na pesquisa das Representações Sociais. In: JOVCHELOVITCH,

Sandra; GUARESCHI, Pedrinho (Org.). Textos em Representações Sociais. Petrópolis (RJ): Vozes, 1994.

De outro modo, a Figura 1 constitui uma representação da distribuição de todo o conjunto das fontes de referência listadas, em relação à sua antiguidade (quadriênio de publicação).

Considerando os nove quadriênios aí representados, verifica-se um contexto gráfico em que se divisam quatro períodos.

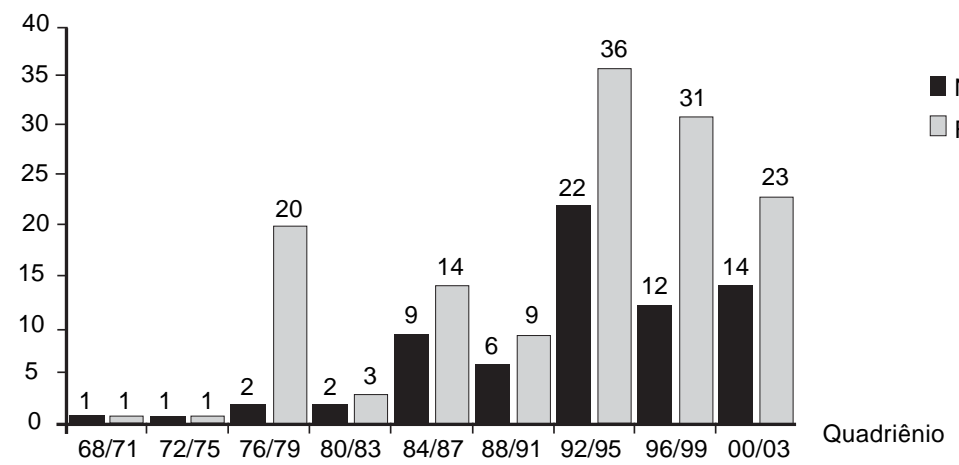

Número de fontes identificadas

Freqüência de referenciamento

Figura 1. Número de fontes da Teoria das Representações Sociais e respectiva freqüência com que se encontram referenciadas em 45 artigos que as utilizam para embasamento teórico-metodológico, considerando o quadriênio em que ocorreram suas publicações. 
O primeiro, de 1968 até 1975, corresponde à publicação de dois dos textos citados sobre TRS (2,9\%, dos elencados nesse estudo); o segundo, de 1976 a 1983, correspondendo ao período de publicação de quatro textos referenciados (5,8\% dos textos); o terceiro período, de 1984 a 1981, corresponde ao período de publicação de 15 textos citados (21,7\% dos textos de referência), e o mais recente, de 1992 a 2003, que corresponde ao da publicação de 48 textos (70\% dos utilizados como referência sobre TRS no elenco dos artigos analisados). Dessa forma, desenha-se uma evolução que se aproxima de uma progressão geométrica no que se refere ao número de textos utilizados, em correspondência àqueles quatro períodos cronológicos. Por esse mesmo gráfico, verifica-se que, para os dois textos do primeiro período, incide uma referência para cada um deles. Para os quatro textos do segundo período, 23 referências foram encontradas. Para os 15 textos do terceiro período, 23 referências foram encontradas e aos 48 textos originários do quarto período, corresponde um total de noventa referências.

Por ordenamento da freqüência com que foram referenciados, identificam-se, na Tabela 1, os seguintes textos como os mais utilizados: A representação social da psicanálise, de Moscovici (1978), fonte referenciada em 42\% dos artigos; Núcleo central das representações sociais, de Sá (1996), texto referenciado em 20\% dos artigos; As representações sociais: um domínio em expansão, de Jodelet (2001), referenciado em 18\% dos artigos; Pratiques sociales et représentations, de Abric (1994, 1997), referenciado em 16\% dos artigos; A abordagem estrutural das representações sociais, de Abric (1998, 2000), referenciado em 13\% das publicações, e A construção do objeto de pesquisa, de Sá (1998), em 11\% dos artigos. Os demais textos, também relacionados na Tabela 1, encontram-se citados em menos de $10 \%$ dos artigos.

Por outro lado, considerando que $60 \%$ das 138 referências sobre a TRS, computadas nos 45 artigos, recaem sobre textos de quatro autores - uma comparação numérica referente a como se dá a presença dos mesmos é destacada na Tabela 2.

Tabela 2. Freqüência absoluta (n) e relativa (\%) com que são referenciados autores mais citados sobre TRS, no elenco de 45 artigos de pesquisa empírica sobre Representações Sociais em Saúde.

\begin{tabular}{lccccc}
\hline \multicolumn{1}{c}{ INDICADOR } & \multicolumn{3}{c}{ AUTORES } & & \\
\cline { 2 - 6 } & $\begin{array}{c}\text { ABRIC } \\
\mathbf{n}(\%)\end{array}$ & $\begin{array}{c}\text { JODELET } \\
\mathbf{n}(\%)\end{array}$ & $\begin{array}{c}\text { MOSCOVICI } \\
\mathbf{n}(\%)\end{array}$ & $\begin{array}{c}\text { SÁ } \\
\mathbf{n}(\%)\end{array}$ & $\begin{array}{c}\text { A + J + M + S } \\
\mathbf{n}(\%)\end{array}$ \\
\hline Número de artigos que referenciam um ou mais textos do autor & $11(24,4)$ & $17(37,8)$ & $22(48,9)$ & $14(31,1)$ & $/$ \\
Número de textos referenciados & $6(8,7)$ & $10(14,5)$ & $7(10,1)$ & $3(4,3)$ & $26(37,7)$ \\
Número total de entradas em listas de referência & $18(13,0)$ & $19(13,8)$ & $28(20,3)$ & $18(13,0)$ & $83(60,1)$ \\
\hline
\end{tabular}

Ainda de acordo com a Tabela 2, por ordenamento decrescente das freqüências apontadas, verifica-se que, quanto ao número de artigos que referenciam aqueles autores, estabelece-se a seguinte hierarquia: Moscovici - Jodelet - Sá - Abric; em função do número de textos referenciados: Jodelet - Moscovici - Abric - Sá, e quanto ao número de vezes que o autor entra em lista de referências: Moscovici - Jodelet - Abric e Sá.

\section{Considerações}

O critério da utilização de revistas indexadas, para nelas localizar artigos baseados na TRS, foi estabelecido com base na premissa de abranger veículos de publicação que, além da seleção qualitativa a que submetem seus artigos, correspondam à expectativa de coerência quanto às 
áreas que são referidas como de sua cobertura e interesse. Embora a base Lilacs seja, possivelmente, a que indexa o maior número de periódicos brasileiros de Ciências da Saúde, deve-se ressalvar a possibilidade de que o presente estudo não tenha abrangido todo e qualquer trabalho empírico fundamentado na TRS e publicado no período aqui considerado. Para isso, também contribuíram diversos aspectos determinantes do insucesso quanto às tentativas de obtenção de vários periódicos. Entre estes estão as limitações quanto a sua distribuição e os problemas editoriais que sofrem, mesmo os periódicos já indexados.

Por outro lado, a não contemplação de revistas de subáreas da Medicina, exceto as que também são referidas à Saúde Coletiva, relaciona-se à pequena probabilidade de ocorrência de artigos baseados na TRS, segundo avaliação prévia feita em uma amostra representativa do grande número dessas revistas listadas na base Lilacs.

Nesse contexto, a busca por trabalhos empíricos fundamentados na TRS, abrangendo um elenco de 46 periódicos nacionais indexados da área de Saúde, propiciou a obtenção de 45 artigos de pesquisa localizados em 19 daquelas revistas. Ressalta-se que os de Enfermagem e de Saúde Pública/Coletiva foram os que mais contemplam publicações baseadas nas TRS. Aliás, a maior presença de profissionais/pesquisadores vinculados a esses campos, como autores de trabalhos com RS em Saúde, conforme constatado por Rummler e Spínola (2005), é uma das características da aderência a essa metodologia aplicada à investigação científica na abordagem de questões que articulam aspectos desta área com as Ciências Sociais.

De outro modo, o que acima é referido em relação à Enfermagem, pode-se associar com o que destacam Marques et al. (2004) sobre a produção, entre 1979 e 2000, de 72 trabalhos, entre dissertações e teses de doutorado, que abordam RS. Com esse fato, ocorrido em apenas um centro de pesquisas em enfermagem, aqueles autores ilustram a citação de Sá (1998) de que o campo de estudos com representações sociais encontra-se em expansão desde a década de 1980, tanto na área da psicologia social como da educação, enfermagem e serviço social.

Já no que se refere aos dados pertinentes às publicações em Saúde Pública/Coletiva, os estudos podem estar relacionados à busca de representações sociais como percurso possível de responder ao que observa Smeke (1993, p.45), quanto ao fato de que "a descrição e análise de experiências de articulação entre agentes de assistência à saúde e a população são fundamentais para a definição de projetos que, efetivamente, encontrem resposta junto à sociedade, tanto na qualificação do serviço em si quanto em relação ao aspecto pedagógico".

Quanto ao levantamento das obras de referência, é oportuno considerar que a identificação de textos sobre a TRS, por meio da análise de citações, não leva necessariamente à constituição da bibliografia existente sobre a mesma, na medida em que, por esse caminho, apenas se revelam impactos literários, o que, por outro lado, se coaduna com um dos objetivos do presente estudo.

Entretanto, a listagem resultante da primeira etapa do trabalho, alcançando a identificação de 69 fontes das quais provieram asserções teóricas a respeito de representações sociais, citadas nos artigos examinados, pode constituir um elemento facilitador da busca a essas fontes por parte de eventuais interessados. Considere-se que a lista dessas fontes relaciona livros, ou apenas capítulos de livros, ou artigos, independente do tipo de trabalho abordado. Edições distintas, ou traduções de uma mesma obra, foram discriminadas em razão de não só constituírem entes bibliográficos diversos, como também visando alternativas para sua localização e acesso. 0 qualificativo de fonte teórica é considerado em relação à natureza do conteúdo citado, pois estudos com abordagem empírica, fundamentados na TRS, tanto podem referir pressupostos teóricos da mesma, quanto neles serem produzidas teorizações. Tal aspecto é próprio de estudos com RS, que, conforme Sá (1996), conjugam fenômenos, conceitos e teorias.

Quanto à ocorrência de citações dessas fontes nos artigos aqui analisados, verifica-se um quadro geral em que, por um lado, há convergência da busca de fundamentações por parte dos pesquisadores empíricos, direcionada para algumas obras, e, por outro lado, uma diversificação na utilização de outras fontes de referência.

O primeiro aspecto é evidenciado pelo fato de que 12 das 69 fontes acumulam mais da metade das referências. Para diversificação, contribui o fato de que a maioria das demais fontes é citada 
em apenas um artigo, assim como há autores citados em apenas um trabalho, conforme aponta a Tabela 1. Por outro lado, é possível que a evolução da diversificação, à semelhança de uma progressão geométrica estabelecida pela sucessão da origem cronológica das fontes (periódicos), retrate a existência de um crescente envolvimento de autores que contribuem para a afirmação desse paradigma.

Pela relação existente entre a freqüência de referenciamento e o número de textos aos quais correspondem essas referências, considerando a data de sua publicação (Figura 1), aparecem como destaques os quadriênios 1976/1979 e 1996/1999. Confrontados esses quadriênios com o que indica a Tabela 1, verifica-se que o destaque do primeiro é atribuível a apenas um texto, o de Moscovici, datado de 1976. Para o destaque do quadriênio 96/99, contribui a recorrência a três textos: de Sá, datado de 1996; de Abric, com publicação em 1998 e 2000, e outro de Sá, datado de 1998. Por outro lado, como publicação mais recente, destaca-se; entre as obras do quadriênio 2000/2003; a recorrência feita ao texto "As representações sociais: um domínio em expansão", de autoria de Jodelet (2001).

Tais aspectos podem estar associados ao que Arruda (2002b) e Pavarino (2004) mencionam, baseados em texto de Sá (1998), que identifica o desenvolvimento, dentro da TRS, de "correntes teóricas complementares": a de Jodelet, mais próxima à teoria original de Moscovici, e por alguns denominada, segundo Arruda (2002a, p.223), de "abordagem processual, pela ênfase justamente nos processos constituintes das Representações Sociais"; e a de Jean-Claude Abric, que enfatiza a dimensão cognitivo-estrutural das RS, ou, mais conhecidamente, a Teoria do Núcleo Central. Entretanto, não foi flagrada, nos artigos examinados, citação a texto que tivesse Wilhem Doise como autor, o que representaria recorrência à fonte nuclear de uma terceira corrente, a da denominada Escola de Genebra que, de acordo com Arruda (2002a, p.223), tem "grande interesse pelas determinantes psicológicas da representação social, e pelos princípios organizadores contidos nas Representações Sociais", aspectos mais presentes em publicações classificadas como de Ciências Humanas, ou de Ciências Sociais, aqui não incluídas. Por outro lado, o perfil geral de recorrência a uma diversidade de autores, correspondente a uma apropriação teóricometodológica compatibilizada nos trabalhos às respectivas questões temáticas, abrangendo o aporte de textos de reflexão mais pontuais, embora presentemente menos citados, parece desenhar um panorama de crescente aderência tanto empírica quanto teórico-literária do que (parafraseando um título antes citado), representaria a TRS para a pesquisa em Saúde: um caminho em expansão.

\section{Referências}

ARRUDA, A. Novos significados da saúde e as representações sociais. Cad. Saúde Colet., v.10, n.2, p.215-27, 2002a.

Teoria das representações sociais e teorias de gênero. Cad. Pesqui., n.117, p.127-47, 2002b.

ASSOCIAÇÃO BRASILEIRA DE NORMAS TÉCNICAS - ABNT. NBR 6023: Informação e documentação, Referências, Elaboração. Rio de Janeiro, 2002.

BIREME. Sistema Latino-Americano e do Caribe de Informação em Ciências da Saúde. BVS - Biblioteca Virtual em Saúde. Lilacs: periódicos indexados - lista por país. Disponível em: <http://www.bireme.br/abd/P/listapais.htm>; $<$ http://bvsmodelo.bvsalud.org/site/lilacs/P/diretorio.htm>. Acesso em: 16 jun. 2003.

BRICEÑO-LEÓN, R. Tendências da pesquisa em Ciências Sociais e Saúde na América Latina: uma perspectiva sociológica. In: CANESQUI, A.M. (Org.). Ciências Sociais e Saúde. São Paulo: Hucitec/Abrasco, 1995. p.31-47.

CARDOSO, G.; ARRUDA, A. As representações sociais da soropositividade entre as mulheres e a adesão ao tratamento. Cad. Saúde Colet, v.11, n.2, p.183-99, 2003.

CARVALHO, V. Acerca de las bases teóricas, filosóficas, epistemológicas de la investigación científica. El caso de la Enfermería. Rev. Latinoam. Enferm., v.16, n.6, p.807-15, 2003.

FRANCO, M.L.P.B. Representações sociais, ideologia e desenvolvimento da consciência. Cad. Pesqui., v.34, n.121, p.169-86, 2004. 
MARQUES, S.C.; OLIVEIRA, D.C.; GOMES, A.M.T. Aids e representações sociais: uma análise comparativa entre subgrupos de trabalhadores. Psicol. Teor. Prat., ed. especial, p.91-104, 2004.

MINAYO, M.C.S. O desafio do conhecimento. 8.ed. São Paulo: Hucitec, 2004.

PAVARINO, R.N. Teoria das representações sociais: pertinência para as pesquisas em comunicação de massa. Comun. Espac. Público, v.7, n.1/2, p.128-41, 2004.

RUMMLER, G.; SPÍNOLA, A.W. Tendências temático-profissionais da pesquisa brasileira com representações sociais em Saúde. O Mundo da Saúde, v.29, n.2, p.129-34, 2005.

. Identificação e procedência de periódicos nacionais referentes à temática de saúde pública ou saúde coletiva, editados entre 1998 e 2003. Cad. Saúde Pública, v.20, n.4, p.1041-9, 2004.

SÁ, C.P. A construção do objeto de pesquisa em representações sociais. Rio de Janeiro: Eduerj, 1998.

. Núcleo central das representações sociais. Petrópolis: Vozes, 1996.

SMEKE, E.L.M. Descobrindo percursos para a pesquisa em saúde no trato com o movimento social. Cad. Saúde Pública, v.9, n.1, p.39-47, 1993.

SPINK, M.J. O conceito de representação social na abordagem psicossocial. Cad. Saúde Pública, v.9, n.3, p.300-8, 1993.

Observa-se, em atividades que levam à formulação de políticas, serviços, pesquisa e educação, que nem sempre os processos de obtenção ou utilização de dados empíricos encontram-se ancorados em bases teóricas. Observam-se, também, informações teóricas desvinculadas da experiência empírica. Por outro lado, no âmbito da publicação científica em Saúde, nota-se a crescente presença da abordagem qualitativa e, também, a crescente adesão de pesquisadores que fundamentam sua metodologia na Teoria das Representações Sociais (TRS). No presente estudo, por meio de análise de citações relacionadas a 45 relatos de trabalhos empíricos embasados na TRS, foram identificadas 69 fontes bibliográficas dessa teoria. Formula-se uma listagem dessas fontes com o intuito de favorecer uma eventual busca por parte de estudantes ou pesquisadores interessados. Traça-se, também, um perfil bibliométrico referente a diversos aspectos das fontes identificadas e sua ocorrência no conjunto analisado.

PALAVRAS-CHAVE: Pesquisa qualitativa. Representações sociais. Teoria das Representações Sociais. Saúde. Bibliometria.

Theoretical sources of social representations: a bibliometric profile of texts cited in Brazilian scientific journals on Health

In activities leading to the formulation of policies and services, in either research or education, it can be seen that the processes of acquisition and utilization of empirical data are not always anchored on theoretical foundations. At the same time, theoretical formulations appear as alienated from experience. On the other hand, a qualitative approach and an increasing adherence of researchers basing their methodology on the Social Representations Theory (SRT) seem to be more present in Health scientific publications. Through the analysis of citations applied to 45 reports of SRT-based empirical work, 69 bibliographical sources of that theory have been identified. This paper presents a list of such sources as a way of favoring the search by students or researchers concerned with the subject. Also, a bibliometrical profile was drawn of various aspects of those sources and of their recurrence within the group under analysis.

KEY WORDS: Qualitative research. Social representations. Social Representations Theory. Health. Bibliometrics.

Fuentes teóricas sobre representaciones sociales: un perfil bibliométrico de textos citados en revistas científicas brasileñas del área de salud

En las actividades que conducen a la formulación de las políticas, de los servicios, de la investigación y de la educación no siempre los procesos de adquisición o uso de datos empíricos están anclados en bases teóricas. Se observan también informaciones teóricas enajenadas de la experiencia empírica. Por otra parte, en el campo de las publicaciones científicas referentes a la salud, se nota la presencia creciente de metodologías de planteamiento cualitativo y también la creciente adherencia de investigadores que fundamentan su método en la Teoría de las Representaciones Sociales (TRS). Por medio de análisis de citaciones relacionadas a 45 relatos empíricos con embasamiento en la TRS, se identifican 69 fuentes bibliográficas de esta teoría. Este artículo presenta una lista de tales fuentes para favorecer su búsqueda por parte de estudiantes o investigadores interesados. También se traza un perfil bibliomático referente a diversos aspectos de las fuentes identificadas y de su ocurrencia en el conjunto analizado.

PALABRAS CLAVE: Investigación cualitativa. Representaciones sociales. Teoría de las Representaciones Sociales. Salud. Bibliometría. 